\title{
VARIÁVEIS CLIINICAS E MACROSCÓPICAS QUE INFLUENCIAM O PROGNÓSTICO
DO CARCINOMA COLORRETAL
}

\author{
Samer FARHOUD ${ }^{1}$, Sansom Henrique BROMBERG ${ }^{2}$, \\ Elci BARRETO ${ }^{3}$ e Antonio Cláudio GODOY ${ }^{4}$
}

RESUMO - Racional - A evolução paradoxal de aproximadamente $1 / 3$ dos doentes com neoplasias catalogadas nos estádios B e C de Dukes mostra ser desejável a adição de outros critérios prognósticos. O valor prognóstico de parâmetros clínicos e macroscópicos da neoplasia colorretal é pouco estudado e os relatos da literatura são controversos, razão pela qual procurouse avaliar a importância prognóstica desses parâmetros. Casuística e métodos - Foram estudados 320 enfermos com câncer colorretal submetidos a extirpação curativa, com idade mediana de 58 anos, sendo 199 (62,2\%) do sexo feminino e 121 (37,8\%) do masculino. Os doentes foram distribuídos em três grupos etários: com menos de 40 anos, entre 40 e 60 anos e com mais de 60 anos. Os tumores foram distribuídos em três segmentos do intestino: cólon direito, cólon esquerdo e reto. As neoplasias foram classificadas em pequenas, iguais ou menores de $35 \mathrm{~mm}$ e grandes, com diâmetro maior de $35 \mathrm{~mm}$. Foram classificadas em exofíticas, quando caracterizadas por crescimento luminal e endofíticas, quando de crescimento intramural. O envolvimento da circunferência intestinal no local da neoplasia foi considerado como parcial ou total. Resultados - Dos 320 doentes, 22 (6,9\%) tinham menos de 40 anos, $159(49,7 \%)$ de 40 a 60 anos e $139(43,4 \%)$ apresentavam idade maior de 60 anos. Setenta e três $(22,8 \%)$ das neoplasias situavam-se no cólon direito, $130(40,6 \%)$ no cólon esquerdo e 117 (36,6\%) no reto. Quanto ao tamanho, $280(87,5 \%)$ eram grandes e $40(12,5 \%)$ pequenas; lesões exofíticas predominaram sobre as endofíticas - 173 $(54,1 \%) \times 147(45,9 \%)$. Maior número de tumores apresentou envolvimento total da circunferência intestinal - 216 $(67,5 \%)$, enquanto $104(32,5 \%)$ apresentaram envolvimento parcial. A sobrevivência de 5 e 10 anos dos doentes não foi influenciada pela idade e sexo dos mesmos, nem pelo local e tamanho das neoplasias. Lesões exofíticas propiciaram maior sobrevivência a seus portadores $-65,9 \%$, quando comparadas às endofíticas $-49,0 \%$. A sobrevida dos doentes com lesões envolvendo parcialmente a circunferência intestinal foi maior que a daqueles com envolvimento total - 72,1\% x 51,4\%. Conclusioes - Isoladamente, variáveis clínicas não influenciaram o prognóstico dos doentes. Das variáveis macroscópicas, a forma e o envolvimento neoplásico da circunferência do intestino influenciaram a sobrevida dos mesmos, mostrando-se capazes de contribuir na identificação de subpopulações de doentes com maior ou menor risco prognóstico.

DESCRITORES - Neoplasias colorretais, diagnóstico. Carcinoma, diagnóstico.

\section{INTRODUÇÃO}

A sobrevivência dos portadores de neoplasias colorretais, após procedimento cirúrgico curativo, é dependente de uma série de fatores, entre os quais se destaca o estudo anatomopatológico da neoplasia extirpada.

O nível de penetração neoplásica na parede intestinal e o comprometimento ou não dos linfonodos, variáveis que compõem a classificação de Dukes, desempenham importante papel no prognóstico dos portadores destas neoplasias. No entanto, aproximadamente $1 / 3$ dos doentes submetidos a exérese curativa dos tumores catalogados no estádio B e C apresentam evolução paradoxal, contrastando com os demais doentes da mesma categoria. Em conseqüência, grande é a demanda de outros parâmetros que, somados aos citados, ampliem o percentual prognóstico desses doentes.

Algumas variáveis clínicas e morfológicas macroscópicas parecem capazes de exercer tal influência. Entre as primeiras

\footnotetext{
Trabalho realizado na ${ }^{1}$ Pós-Graduação, no ${ }^{2}$ Serviço de Anatomia Patológica e no ${ }^{3}$ Serviço de Gastroenterologia Cirúrgica do Instituto de Assistência Médica ao Servidor Público Estadual (IAMSPE) e Hospital do Servidor Público Estadual - Francisco Morato de Oliveira (HSPE-FMO), São Paulo, SP.

Endereço para correspondência: Dr. Sansom Henrique Bromberg - Rua Conselheiro Brotero, 1505 - conj. 51 - 01232-010 - São Paulo, SP. e-mail: cedep@iamape.saude.sp.gov.br
} 
merecem especial referência a idade, o sexo e o local da neoplasia, enquanto o tamanho, a forma e o envolvimento da circunferência do intestino no local onde a lesão está assestada são as macroscópicas de maior interesse. No entanto, poucos investigadores dedicaram-se ao estudo dessas variáveis ${ }^{(2,8,14,29)}$.

O câncer colorretal é doença predominante da população idosa, que vem aumentando paulatinamente e deve aumentar ainda mais, frente a maior expectativa de vida ${ }^{(6)}$. A sobrevida global dos idosos é geralmente menor que a dos mais jovens, devido a maior morbidade $\mathrm{e}$ mortalidade cirúrgica ${ }^{(21)}$. Outros estudiosos acreditam que, superados os problemas pós-operatórios, a sobrevivência de idosos seria próxima à dos grupos de menor idade ${ }^{(13)}$.

Por outro lado, o acentuado aumento da incidência do carcinoma colorretal no cólon direito, não foi observado em países não industrializados ${ }^{(10,11,19,31)}$. Tampouco a maior incidência destes tumores no sexo feminino foi constatada universalmente ${ }^{(27)}$.

Na maior parte dos estudos, o tamanho dos tumores não ofereceu nenhum subsídio prognóstico ${ }^{(4,28,33)}$

Tumores exofíticos - polipóides e vegetantes - tenderiam a oferecer melhor prognóstico; o tipo mais agressivo e, portanto, de pior prognóstico, estaria associado às formas de crescimento intramuralinfiltrativa e ulcerada, mesmo quando pequenos ${ }^{(13)}$.

Aparentemente, à medida que o tumor cresce no envolvimento circular do intestino, o número de metástases nos linfonodos aumenta, a recidiva é mais freqüente e a sobrevivência diminui ${ }^{(14,23,35)}$.

Interessados no comportamento de variáveis capazes de influenciar o prognóstico de portadores de carcinomas colorretais e frente a essas controvérsias, decidiu-se estudar o papel prognóstico de alguns parâmetros clínicos - sexo, idade, local-e morfológicos macroscópicos da neoplasia colorretal - tamanho, forma e envolvimento da circunferência intestinal -, visando identificar subgrupos de enfermos propícios de seguimento mais ou menos intensivo.

\section{CASUÍSTICA E MÉTODOS}

Foram estudados 320 doentes com carcinoma colorretal submetidos a tratamento cirúrgico no Serviço de Gastroenterologia Cirúrgica do Hospital do Servidor Público Estadual - Francisco Morato de Oliveira, São Paulo, SP, entre 1964 e 1980. Todos os pacientes foram seguidos até o óbito ou no mínimo por 5 anos. O maior período de acompanhamento foi de 295 meses e o menor de 3 meses, com média de $80,82 \pm 63,90$ meses.

Os procedimentos operatórios foram realizados por pequeno número de cirurgiões com a mesma formação, empregando idêntica padronização cirúrgica. Em todos os casos a equipe operatória considerou curativa a extirpação da neoplasia. Este critério curativo deve ser entendido como ausência de crescimento do tecido neoplásico à distância do tumor primitivo, inexistência de neoplasia residual visível e ausência de comprometimento das margens da peça extirpada constatada pelo estudo anatomopatológico.

As características macroscópicas das neoplasias foram colhidas dos relatórios cirúrgicos e dos laudos anatomopatológicos originais. A evolução clínica dos doentes foi obtida pela revisão dos prontuários hospitalares, por entrevistas ambulatoriais com os mesmos ou seus familiares e por telefone ou correspondência.

A idade dos doentes variou de 28 a 86 anos, com média de 58,10 $\pm 12,06$ anos e mediana de 58 anos. Os doentes foram agrupados em três faixas etárias, a saber: os com menos de 40 anos, aqueles entre 40 e 60 anos e os maiores de 60 anos.

As neoplasias foram classificadas de acordo com o tamanho, medido antes da fixação da peça extirpada, em dois grupos: pequenas, quando mediam em seu maior diâmetro $35 \mathrm{~mm}$ ou menos, e grandes, com diâmetro acima de $35 \mathrm{~mm}$. A média de tamanho dos 320 tumores foi de $62,8 \mathrm{~mm}$, variando de 10 a $220 \mathrm{~mm}$.

No tocante à forma, os tumores foram classificados em exofíticos, quando se projetavam para a luz do órgão, pouco progredindo para o interior da parede intestinal. Aqueles que progrediam para o interior da parede, preferencialmente tumores ulcerados e infiltrativos, foram denominados endofíticos.

O grau de envolvimento circular do segmento intestinal pela neoplasia foi observado no nível de maior crescimento da mesma, sendo classificado em parcial e total.

Para análise dos resultados foi adotado nível de significância de 5\% $(\alpha=0,05)$. Aplicou-se o teste do qui-quadrado corrigido para continuidade, segundo Yates, para tabelas 2 × 2, com o objetivo de analisar as associações entre as variáveis estudadas. Quando presentes mais de duas variáveis (tabelas 2 x N), efetuou-se o teste do qui-quadrado comum. Com a finalidade de obter as sobrevivências dos doentes até 10 anos e comparálas às de 5 anos, empregou-se o método de Kaplan-Meyer. A análise dos valores obtidos foi realizada pelo "log rank test".

\section{RESULTADOS}

Maior incidência de doentes ocorreu no sexo feminino e na faixa etária entre 40 e 60 anos; os tumores predominaram nas regiões distais do intestino. Constatou-se nítido predomínio dos tumores de maior proporção e a maioria das neoplasias apresentou comprometimento completo da circunferência do segmento intestinal em que se assestavam; a distribuição quanto à forma foi eqüitativa. Os dados relativos aos parâmetros clínicos e macroscópicos podem ser analisados na Tabela 1.

A sobrevida de 5 anos dos 320 doentes com câncer colorretal que integraram este estudo foi de $58,1 \%$.

Os valores relacionados à sobrevida de 5 anos e seu grau de significância, segundo as variáveis estudadas, podem ser analisados na Tabela 2. 
TABELA 1- Distribuição das variáveis clínicas e macroscópicas em 320 doentes com carcinoma colorretal

\begin{tabular}{lcc}
\hline Variáveis & No. & $\%$ \\
\hline Idade & 22 & 6,9 \\
$\quad<40$ & 159 & 49,7 \\
$40-60$ & 139 & 43,4 \\
$>60$ & & \\
Sexo & 199 & 62,2 \\
feminino & 121 & 37,8 \\
masculino & & \\
Local & 73 & 22,8 \\
$\quad$ cólon D & 139 & 40,6 \\
cólon E & 117 & 36,6 \\
reto & & \\
Tamanho & 40 & 12,5 \\
pequeno & 280 & 87,5 \\
$\quad$ grande & & \\
Forma & 173 & 54,1 \\
exofítica & 147 & 45,9 \\
endofítica & & 32,5 \\
Env.circunf. & 104 & 67,5 \\
parcial & 216 & \\
total & & \\
\hline
\end{tabular}

Env. circunf. $=$ envolvimento da circunferência intestinal

TABELA 2 - Sobrevida de 5 anos de 320 doentes com câncer colorretal segundo parâmetros clínicos e macroscópicos

\begin{tabular}{|c|c|c|c|c|}
\hline Variáveis & No. & $>5$ anos & 10 anos & valor de $P$ \\
\hline \multicolumn{5}{|l|}{ Idade } \\
\hline$<40$ & 22 & $72,7 \%$ & $54,7 \%$ & \\
\hline $40-60$ & 159 & $62,3 \%$ & $56,6 \%$ & \\
\hline$>60$ & 139 & $51,1 \%$ & $44,1 \%$ & 0,053 \\
\hline \multicolumn{5}{|l|}{ Sexo } \\
\hline feminino & 199 & $59,3 \%$ & $51,7 \%$ & \\
\hline masculino & 121 & $56,2 \%$ & $50,7 \%$ & 0,668 \\
\hline \multicolumn{5}{|l|}{ Local } \\
\hline cólon D & 73 & $58,9 \%$ & $50,2 \%$ & \\
\hline cólon E & 139 & $63,9 \%$ & $59,2 \%$ & \\
\hline reto & 117 & $51,3 \%$ & 43,4 & 0,134 \\
\hline \multicolumn{5}{|l|}{ Tamanho } \\
\hline pequeno & 40 & $65,0 \%$ & $64,8 \%$ & \\
\hline grande & 280 & 57,1 & $49,3 \%$ & 0,441 \\
\hline \multicolumn{5}{|l|}{ Forma } \\
\hline exofítica & 173 & $65,9 \%$ & $57,4 \%$ & \\
\hline endofítica & 147 & $49,0 \%$ & $44,2 \%$ & 0,003 \\
\hline \multicolumn{5}{|l|}{ Env.circunf. } \\
\hline parcial & 104 & $72,1 \%$ & $60,7 \%$ & \\
\hline total & 216 & $51,4 \%$ & $46,8 \%$ & 0,001 \\
\hline
\end{tabular}

Env. circunf. $=$ envolvimento da circunferência intestinal 
As curvas de Kaplan-Meyer associando as variáveis estudadas e a respectiva sobrevivência estimada de 10 anos dos 320 doentes podem ser analisadas nas Figuras 1 a 6.

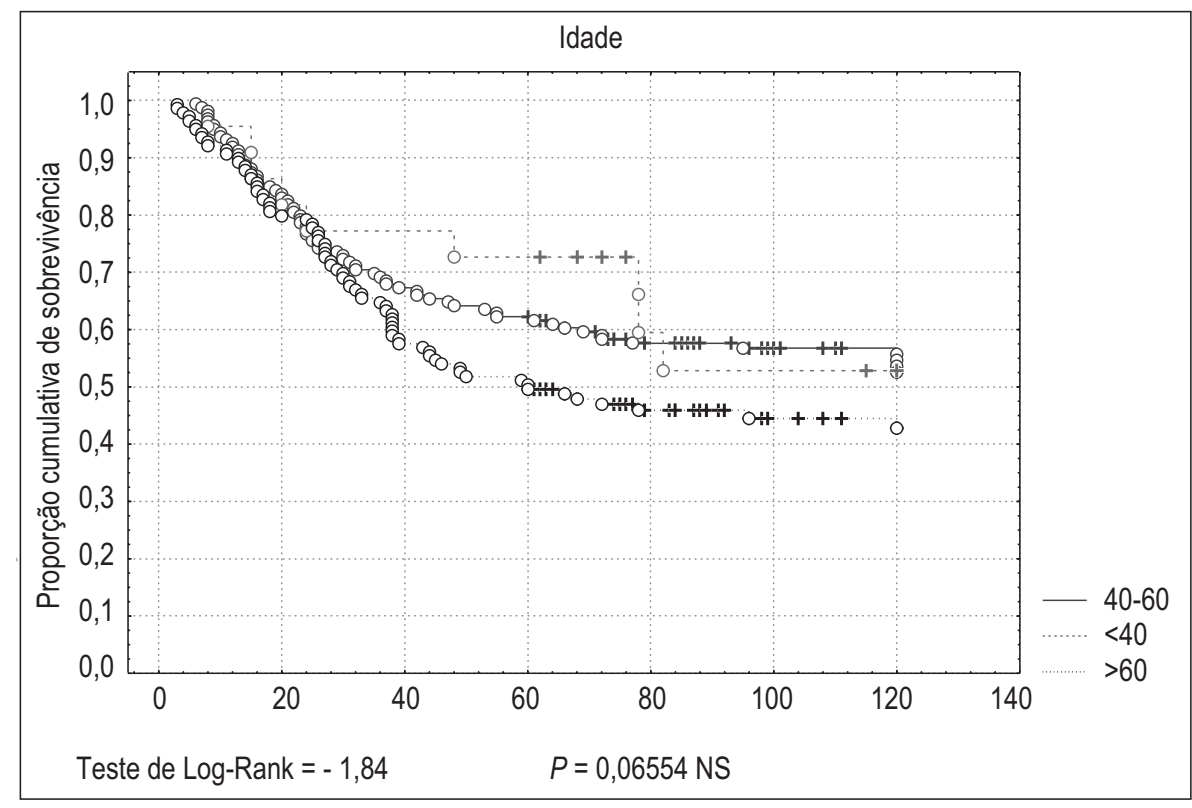

FIGURA 1 - Curvas de sobrevivência estimada de 10 anos de 320 doentes com carcinoma colorretal segundo a idade

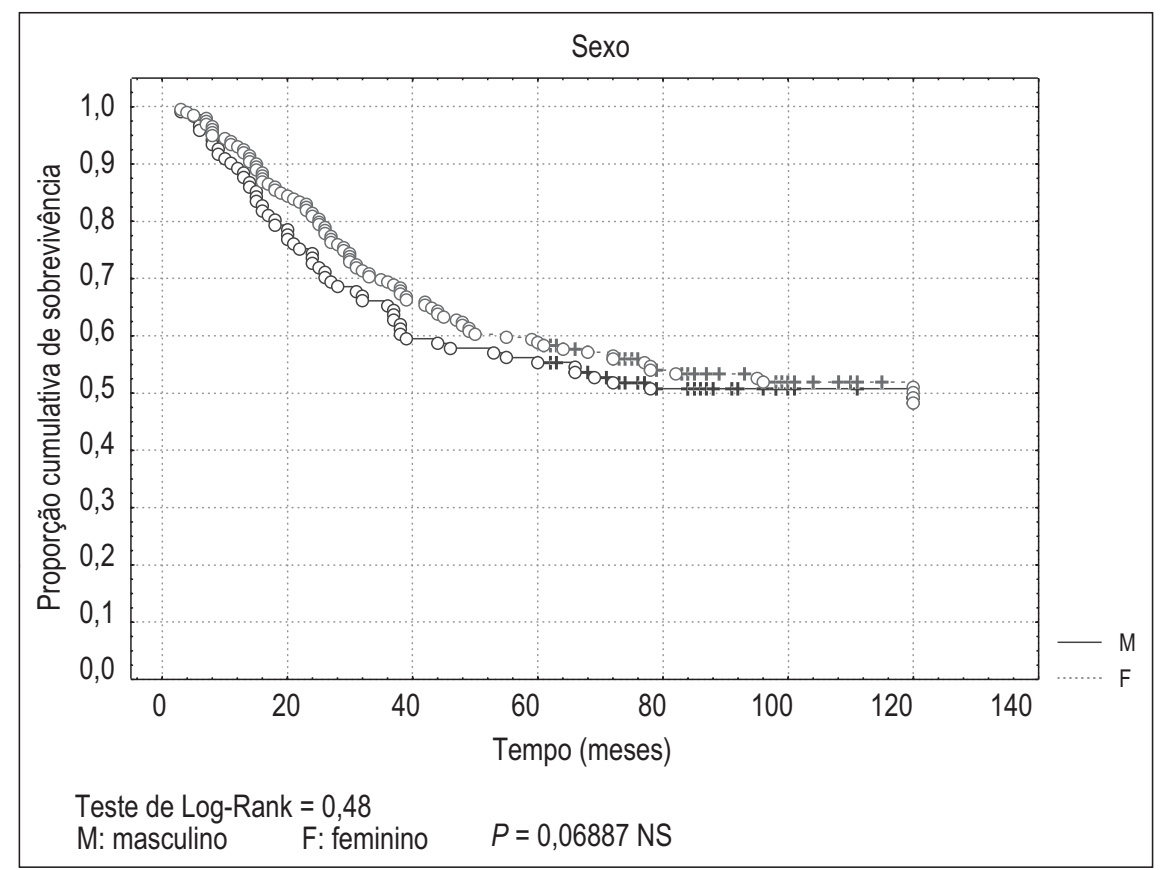

FIGURA 2- Curvas de sobrevivência estimada de 10 anos de 320 doentes com carcinoma colorretal segundo o sexo 


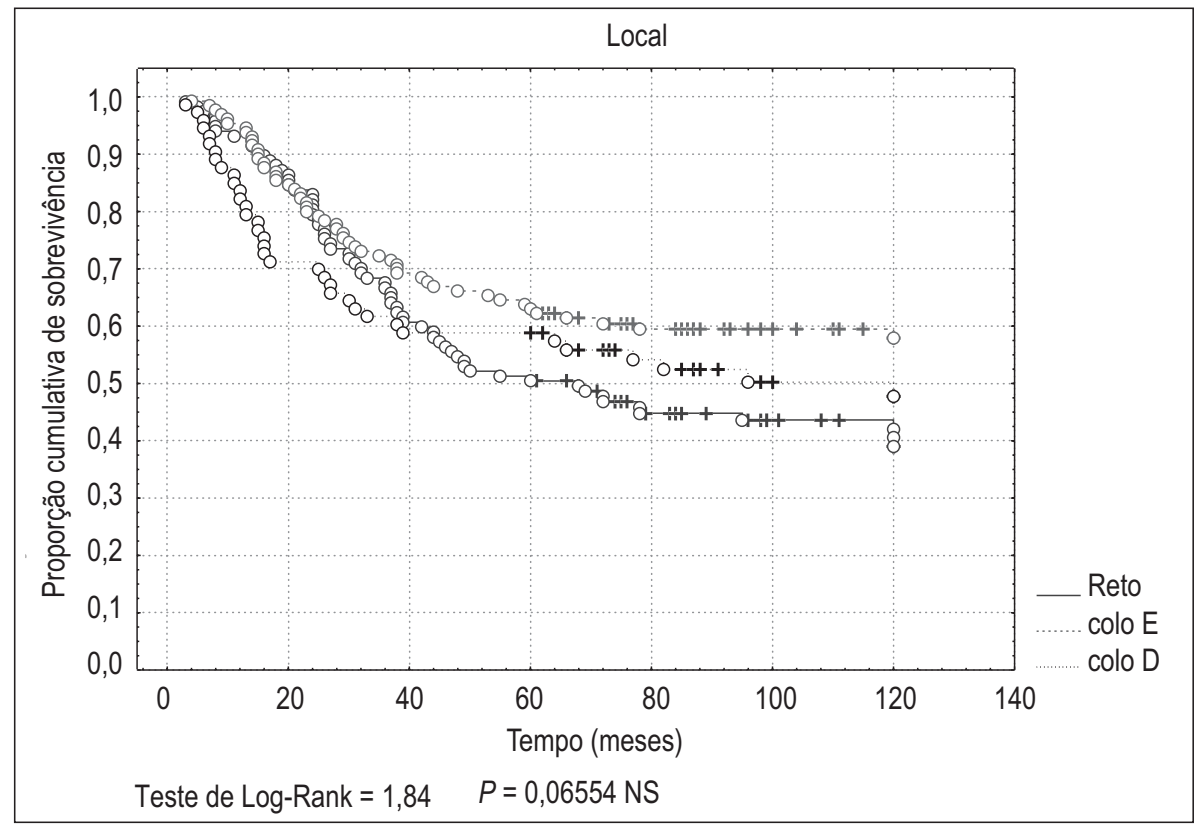

FIGURA 3 - Curvas de sobrevivência estimada de 10 anos de 320 doentes com carcinoma de colo e reto, segundo o local da neoplasia

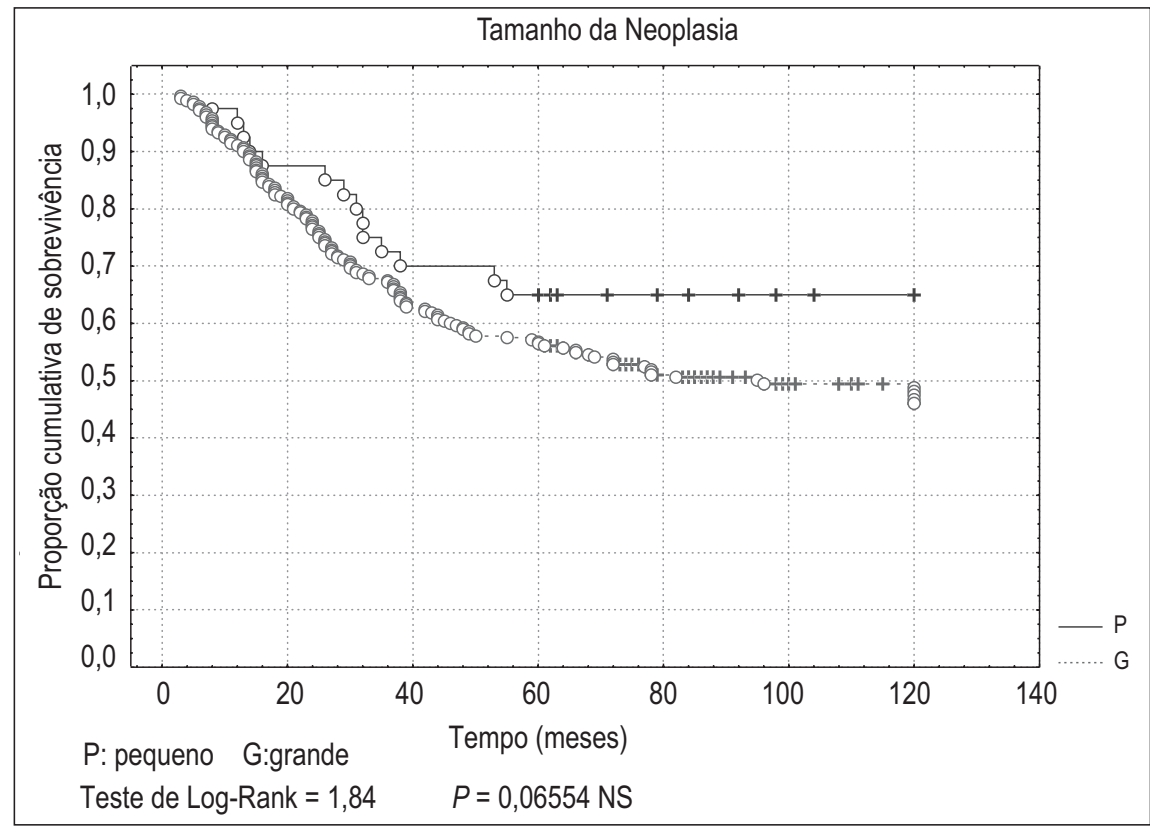

FIGURA 4 - Curvas de sobrevivência estimada de 10 anos de 320 doentes com carcinoma colorretal segundo o tamanho da neoplasia 


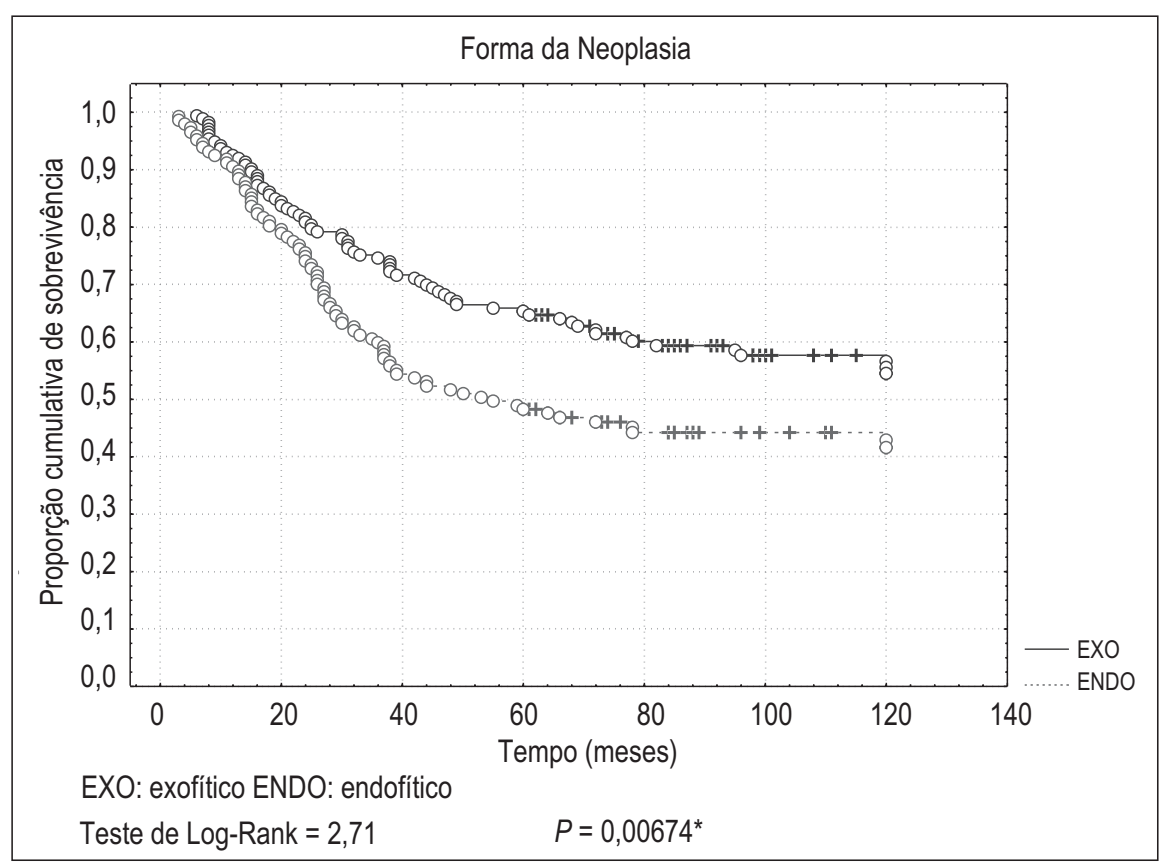

FIGURA 5 - Curvas de sobrevivência estimada de 10 anos de 320 doentes com carcinoma colorretal segundo a forma tumoral

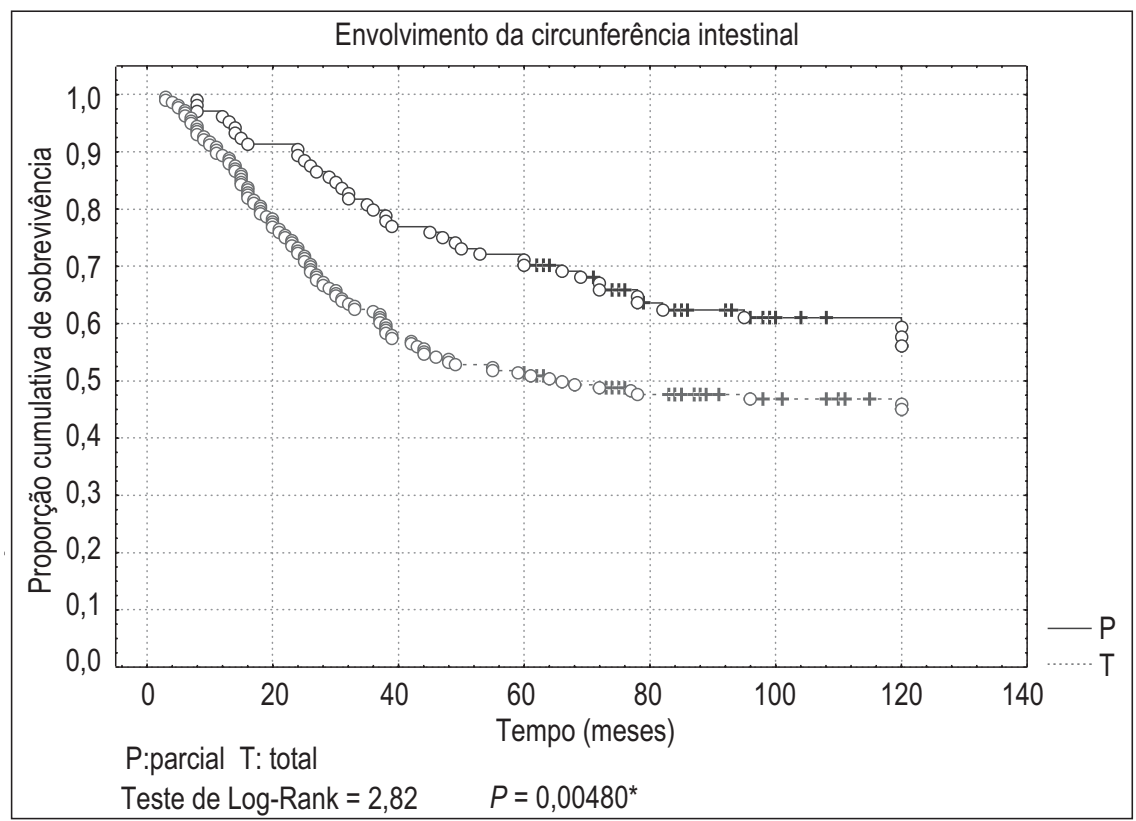

FIGURA 6 - Curvas de sobrevivência estimada de 10 anos de 320 doentes com carcinoma colorretal segundo o grau de comprometimento da circunferência intestinal 


\section{DISCUSSÃO}

Eventos clínicos e macroscópicos merecem ser contemplados em classificações prognósticas, pois quando aliados ao procedimento cirúrgico curativo e aos subsídios fornecidos pela microscopia clássica podem melhor individualizar sub-grupos de doentes com parâmetros semelhantes de sobrevida.

A média de idade dos pacientes deste estudo foi de 58,1 anos, maior que a de 55,6 anos encontrada na revisão de 614 doentes por BRENNER $^{(3)}$, em 1990.

Essas cifras divergem das relatadas em países ou regiões onde a doença tem maior prevalência. Na Escócia, estudo de 312 doentes submetidos a cirurgia curativa ${ }^{(7)}$, relatou média de 66,3 anos, enquanto no Canadá a média foi de 67 anos, em estudo de 524 enfermos ${ }^{(23)}$.

A influência da idade sobre o prognóstico mostra índices conflitantes, quer em estudos com pacientes jovens, quer quando referentes a idosos. Parece cada vez mais difundida a opinião de que a idade, por si só, não se constitui em fator de risco de mortalidade operatória ou de maior tempo de sobrevida, exercendo essa influência o estado fisiológico do paciente ${ }^{(7,18)}$.

Não foram constatadas diferenças significativas no tocante à sobrevida dos doentes quanto à idade. No entanto, a sobrevida no grupo com menos de 40 anos foi a mais expressiva, atingindo índice de $72,7 \%$ comparado a $62,3 \%$ da população entre 40 e 60 anos (Tabela 2 ). Esse valor ganha maior amplitude se atentarmos para o fato da sobrevivência global de 5 anos ter sido de $58,1 \%$. Seguramente uma das razões para explicar esse alto índice de sobrevivência é o fato de todos os doentes em estudo terem sido submetidos a exéreses curativas. Tais valores não foram confirmados na sobrevivência estimada aos 10 anos (Figura 1).

$\mathrm{Na}$ literatura a sobrevida de 5 anos em idosos variou de $22,4 \%$ a $56,0 \%{ }^{(6,18,19)}$.

Dos 186 doentes deste estudo com sobrevivência maior de 5 anos, cerca de $71(51,1 \%)$ deles tinham mais de 60 anos (Tabela 2), resultado comparável aos melhores da literatura.

Maiores índices de sobrevivência são geralmente relatados no sexo feminino ${ }^{(4,12,13,16,34)}$.

Interessante e incomum, o relato de $\mathrm{KOCH}$ et al. ${ }^{(15)}$ merece referência. Em estudo de 1.522 doentes, também constataram maior sobrevida no sexo feminino; no entanto, mulheres sem filhos e também as que nunca engravidaram apresentaram sobrevivência semelhante à dos homens.

Neste estudo, embora a sobrevivência de 5 e 10 anos das mulheres tenha sido maior que a dos homens, esses valores não foram significativos.

As razões para essa maior sobrevivência são desconhecidas, sendo geralmente responsabilizados os hormônios femininos ${ }^{(15)}$. No entanto, tumores intestinais situados na pélvis recidivam mais freqüentemente no sexo masculino, sendo atribuído tal fato a maior dificuldade na sua extirpação devido à estreiteza da mesma e a maior proximidade dos órgãos adjacentes naquele sexo ${ }^{(26)}$.

Acentuadas alterações na distribuição dos tumores intestinais vêm sendo observadas nos últimos 50 anos em países industrializados, provocadas pelo aumento da incidência do carcinoma do cólon direito, acompanhadas de decréscimo numérico do retal ${ }^{(11,31)}$.

Estudo analisando a distribuição de 1.694 dessas neoplasias durante os períodos de 1960-1969, 1970-1979 e 1980-1984 mostrou decréscimo final de $15,8 \%$ para neoplasias do reto e aumento de $10,2 \%$ para as do cólon direito ${ }^{(10)}$. Como conseqüência, os tumores localizados nos primeiros e últimos $25 \mathrm{~cm}$ do intestino seriam responsáveis cada qual por $1 / 4$ dos tumores intestinais.

BERNSTEIN et al. ${ }^{(1)}$ mostraram modelo semelhante na distribuição de pólipos colorretais. O tamanho e o número dos pólipos proximais apresentaram relação direta com o aumentar da idade. Nos doentes com menos de 50 anos, o número deles foi de $6,9 \%$ e nos com mais de 80 foi de $29,3 \%$ no cólon direito, ocorrendo fato oposto no reto.

Explicação parcial para justificar essa menor incidência de tumores no cólon distal seria a retirada endoscópica de pólipos benignos daquele segmento e a ação de sais biliares sobre a mucosa do cólon direito ${ }^{(20)}$.

A implicação maior no tocante a essas mudanças da incidência e distribuição do câncer colorretal diz respeito à carcinogênese desses tumores. Tais fatos sugerem uma interrelação entre fatores ligados ao hospedeiro e fatores ligados à etiologia e mecanismos patogenéticos do câncer intestinal, interagindo de modo diferente de acordo com diferentes locais.

No Brasil, constataram-se $18,08 \%$ das lesões no cólon direito, $21,98 \%$ no cólon esquerdo e $57,82 \%$ no reto ${ }^{(3)}$, enquanto neste estudo a distribuição foi de $22,8 \%, 40,6 \%$ e 36,6\%, respectivamente. Aparentemente, os fatores responsáveis pelas alterações na distribuição das neoplasias no intestino ainda não aportaram em nosso meio.

A sobrevivência de 5 anos dos doentes deste estudo com lesões no cólon direito foi de $58,9 \%$, sendo de $63,9 \%$ para os do cólon esquerdo e de $51,3 \%$ para aqueles com lesões retais, semelhantes aos valores encontrados por BRENNER ${ }^{(1)}$, no Paraná. Mais freqüentemente os índices são superiores $-73 \%, 59 \%$ e $76 \%$, respectivamente ${ }^{(31)}$.

Tais proporções foram mantidas na sobrevivência de 10 anos. Na literatura, os relatos sobre a influência do local da neoplasia sobre o prognóstico são contraditórios.

A localização do tumor no cólon direito associou-se a melhor prognóstico em muitos estudos ${ }^{(8,12,13,15,33)}$. No entanto, as lesões proximais do cólon também foram citadas como de igual ou pior prognóstico que as demais ${ }^{(7,25)}$

Poucos estudos mostraram maior sobrevivência para portadores de lesões retais ${ }^{(2,24,25)}$, prevalecendo aqueles considerando-as como de pior prognóstico quando comparadas às dos cólons ${ }^{(8,12,21,24,28,33,34)}$. 
Estudos recentes mostraram sobrevivência entre $74 \%$ e $77,5 \%$ para doentes com lesões situadas no cólon, enquanto para os com lesões retais foi de $62 \%$ e $66 \%$, respectivamente ${ }^{(13,30)}$.

Os melhores índices de sobrevivência deste estudo foram apresentados por enfermos com tumores localizados no cólon esquerdo, mas esses valores não se mostraram significativos, como relatado em vários trabalhos ${ }^{(4,7,24,31)}$.

Os tumores são geralmente diagnosticados em fase avançada da doença e a maior parte deles se apresenta como grandes formações, fato confirmado neste estudo, onde $87,5 \%$ deles eram maiores de $35 \mathrm{~mm}$ (Tabela 1).

A média de tamanho dos 320 tumores foi de $62,8 \mathrm{~mm}$, maior que o da literatura consultada, onde esse valor variou de 43 a $49 \mathrm{~mm}^{(10,31)}$.

O tamanho da neoplasia não influenciou o prognóstico, na maioria das séries relatadas ${ }^{(4,28,30,33)}$. Outros trabalhos constataram menor sobrevivência para portadores de neoplasias de maior tamanho ${ }^{(18,21,29)}$.

Alguns tumores colorretais atingem grandes proporções sem metastizarem $^{(28)}$. Essa aparente contradição encontra explicação com a relação inversa entre volume da lesão, estádio da doença e a sobrevida dos doentes ${ }^{(33)}$.

O tamanho do tumor pode favorecer a maior perda sangüínea. Tumores maiores de $30 \mathrm{~mm}$ associaram-se a menores taxas séricas de hemoglobina, ferro e ferritina, quando comparados aos menores ${ }^{(17)}$.

Lesões de grande tamanho foram ainda associadas à aneuploidia, alteração do DNA geralmente relacionada a pior prognóstico ${ }^{(30)}$. Quando localizadas no reto recoberto por peritônio, associaram-se a maior recidiva local que os menores ${ }^{(25)}$

Embora a sobrevivência de 5 anos dos doentes com pequenos tumores tenha sido maior neste estudo $(65 \%$ x $57,1 \%)$, bem como a de 10 anos $(64,8 \%$ x 49,3\%), esses valores não foram significativos.

O grande interesse pela ausência da participação do tamanho dos tumores no prognóstico, diz respeito à classificação TNM que, no geral, atribui valor de importância ao tamanho da neoplasia.

A tendência entre os estudiosos é classificar os tumores, quanto a forma, em dois grandes tipos: vegetantes ou exofíticos, com crescimento predominantemente luminal e não vegetantes ou endofíticos, de crescimento intramural predominante, representando os tumores ulcerados e infiltrativos. Na prática diária, os tumores ulcerados e vegetantes de grandes proporções compõem a maioria deles, sendo responsáveis por $30,3 \%$ a $85,6 \%$ das neoplasias colorretais ${ }^{(2,14,29,30)}$.

A forma dos tumores influenciou significativamente a sobrevida dos doentes. A sobrevivência dos portadores de lesões exofíticas $65,9 \%$ - foi nitidamente maior que a dos endofíticos - 49,0\% (Tabela 2).

Tais fatos são praticamente constantes na literatura, com múltiplos estudos realçando a maior sobrevivência dos portadores desta forma de neoplasia ${ }^{(2,14,28,29,30,32)}$.
Portadores de carcinomas exofíticos apresentaram menor incidência de metástases na distante medula óssea e menor percentual de recidivas ${ }^{(23)}$. Ao que parece, aspectos de menor agressividade caracterizam tumores que apresentam crescimento luminal preponderante.

As razões do melhor prognóstico destes tumores são desconhecidas, sendo oferecidas múltiplas explicações, algumas bastante engenhosas: menor disseminação vascular, associação ao tipo expansivo de neoplasia, maior resposta do infiltrado linfoplasmocitário ${ }^{(29)}$.

STEINBERG et al. ${ }^{(29)}$ sugeriram ser o sistema imunológico do indivíduo com tumor exofítico mais capaz de reconhecer e controlar os tumores que o correspondente às outras formas tumorais.

Acredita-se também ser essa associação com melhor prognóstico devida ao fato de estarem as lesões protuberantes mais freqüentemente associadas a estádio clínico patológico menos avançado que as demais formas à época do diagnóstico ${ }^{(12)}$.

Nos últimos anos, importantes alterações genéticas foram constatadas nessas formas tumorais, sugerindo serem as mesmas geneticamente distintas, com diferentes fatores etiológicos e de risco ${ }^{5}$, 9). Haveria pelo menos duas diferentes rotas na carcinogênese colorretal ligadas a essas duas formas: a polipóide ou exofítica, caracterizada pela alta freqüência de mutações K ras e a não-polipóide ou endofítica, com baixa freqüência desta mutação ${ }^{(9)}$.

O envolvimento circular do intestino é importante fator de agressividade neoplásica e está associado a pior prognóstico. A neoplasia demanda 6 meses para revestir a quarta parte da circunferência retal e frente ao envolvimento circular, deduz-se estar a neoplasia instalada há mais de 2 anos; o envolvimento de três quadrantes retais pela neoplasia associou-se à presença de tumores que penetravam espaços extra-retais de moderada a grande extensão, ou seja, em estádio III ${ }^{(22)}$.

Estudo de 597 pacientes com carcinomas retais constatou comprometimento total da circunferência em $47,2 \%$ das neoplasias e parcial em $52,8 \%$. A sobrevida de 5 anos foi, respectivamente, de $83 \%$ para os portadores de envolvimento parcial e de $54 \%$ para o total ${ }^{(14)}$.

Tumores mucinosos são os que com mais assiduidade se associaram a maior envolvimento da circunferência intestinal - 71\% contra $33 \%$ dos não-mucinosos ${ }^{(35)}$.

Os índices de comprometimento da circunferência intestinal deste estudo são maiores, indicando estarem as neoplasias há mais tempo instaladas, já que cerca de $216(67,5 \%)$ delas apresentavam envolvimento total (Tabela 1). Dos doentes com neoplasias exibindo envolvimento parcial, 72,1\% vieram a apresentar sobrevivência superior a 5 anos, comparados a 51,4\% com envolvimento total (Tabela 2). Esses valores continuaram presentes quando estimados por Kaplan-Meyer (60,7\% x $46,8 \%$ ). Tais constatações permitem sugerir que essa variável deva constar do relatório patológico a que o espécime extirpado é submetido.

Fundamentalmente este estudo sugere que jovens, desde que operados curativamente, apresentarão sobrevida igual ou maior que os demais pacientes. Sugere também ser de valia o estudo de 
parâmetros macroscópicos, embora no cotidiano pouca importância seja dedicada aos mesmos. Ensaios clínicos em doentes com tumores de crescimento intramural e envolvimento total da circunferência do intestino devem ser estimulados.

Farhoud S, Bromberg SH, Barreto E, Godoy AC. Clinical and macroscopic variables of colorectal carcinoma that influence the prognosis. Arq Gastroenterol 2002;39(3):163-172.

ABSTRACT - Background and Aims - The paradoxical evolution of approximately one third of patients with neoplasms cataloged in Dukes stages $\mathrm{B}$ and $\mathrm{C}$ demonstrates the desirability of utilizing other prognostic criteria that are capable of broadening the information provided by these two important variables. Only a small number of investigators have dedicated themselves to the study of the prognostic value of clinical and macroscopic parameters of colorectal neoplasms, and the results obtained have been shown to be controversial. The principal aim of this work was to evaluate the prognostic importance of these parameters. Patients and Methods - A study was made of 320 patients with colorectal cancer who underwent curative extirpation. They had a median age of 58 years, and there were 199 females (62.2\%) and 121 males (37.8\%). The patients were divided into three age groups: under 40 years old, between 40 and 60 years old and over 60 years old. The tumors were distributed in three intestinal segments: right colon, left colon and rectum. The neoplasms were classified as small (diameter less than or equal to $35 \mathrm{~mm}$ ) and large (diameter greater than $35 \mathrm{~mm}$ ). With regard to their form, they were classified as exophytic, when characterized by luminal growth, and endophytic, when there was intramural growth. The involvement of the intestinal circumference at the site of the neoplasm was considered as partial or total. Results - Of the 320 patients, 22 (6.9\%) were aged under 40 years, 159 (49.7\%) from 40 to 60 years and 139 (43.4\%) presented an age of over 60 years. Seventy-three $(22.8 \%)$ of the neoplasms were located in the right colon, $130(40.6 \%)$ in the left colon and $117(36.6 \%)$ in the rectum. Regarding the size, $280(87.5 \%)$ were large and $40(12.5 \%)$ small; exophytic lesions predominated over endophytic ones $-173(54.1 \%)$ vs $147(45.9 \%)$. A greater number of tumors presented total involvement of the intestinal circumference - $216(67.5 \%)$ - while $104(32.5 \%)$ presented partial involvement. The 5-year survival of the patients was not influenced by their age and sex, or by the location and size of the neoplasms. Exophytic lesions conferred greater survival on their sufferers (65.9\%), in comparison with endophytic lesions (49.0\%). The survival of patients with lesions partially involving the intestinal circumference was greater than for those with total involvement $-72.1 \%$ vs. $51.4 \%$. Conclusions - Clinical variables had no influence on the patients' prognosis. Among the macroscopic variables, the form of the neoplasia and its involvement in the intestinal circumference did influence the patients' prognosis. These last two variables are important data capable of contributing to the identification of patient subpopulations with greater or lesser prognostic risk.

HEADINGS - Colorectal neoplasms, diagnosis. Carcinoma, diagnosis.

\section{REFERÊNCIAS BIBLIOGRÁFICAS}

1. Bernstein MA, Feczko PI, Halpert RD, Simms SM, Ackerman LV. Distribution of colonic polyps: increased incidence of proximal lesions in older patients. Radiology 1985;155:35-40.

2. Brandão O, Sobrinho-Simões MA, Serrão D, Moutinho-Ribeiro M, Azevedo C Prognosis in colorectal carcinoma: a reassessment of the pathologist's role. Pathol Res Pract 1985;180:506-10.

3. Brenner S. Adenocarcinomas colorretais. Análise dos resultados do tratamento cirúrgico em 608 doentes [tese]. Curitiba: Universidade Federal do Paraná; 1990.

4. Chapuis PH, Dent OF, Fisher R, Newland RC, Pheils MT, Smyth E, Colquhoun $\mathrm{K}$. A multivariate analysis of clinical and pathological variables in prognosis after resection of large bowel cancer. Br J Surg 1985;72:698-702.

5. Chiang JM, Chou YHW, Chou TB. K-ras codon 12 mutation determines the polypoid growth of colorectal cancer. Cancer Res 1998;58:3289-93.

6. Coburn MC, Pricolo VE, Soderberg CH. Factors affecting prognosis and management of carcinoma of the colon and rectum in patients more than eighty years of age. J Am Coll Surg 1994;179:65-9.

7. Deans GT, Heatley M, Patterson CC, Moorehead RJ, Parks TG, Rowlands BJ, Spence RAJ. Colorectal carcinoma: importance of clinical and pathological factors in survival. Ann R Coll Surg Engl 1994;76:59-64.

8. Eisenberg B, Decosse JJ, Harford F, Michalek J. Carcinoma of the colon and rectum. The natural history reviewed in 1704 patients. Cancer 1982;49:1131-4.

9. George SMC, Makinen MJ, Jernvall P, Makela J, Vihko P, Kartunnen TJ. Classification of advanced colorectal carcinomas by tumor edge morphology. Evidence for different pathogenesis and significance of polypoid and nonpolipoyd tumors. Cancer 2000;89:1901-9.

10. Gharemani GG, Dowlatshahi K. Colorectal carcinomas: diagnostic implications of their changing frequency and anatomic distribution. World J Surg 1989; $13: 321-5$.

11. Golematis BC, Tzardis PJ, Ahwal JAL, Charitopoulos N, Peveretos P. Site distribution of carcinoma of the large intestine. Retrospective study of 600 cases. Dis Colon Rectum 1989;32:14-6.

12. Halvorsen TB, Seim E. Tumor site: a prognostic factor in colorectal cancer? A multivariate analysis. Scand J Gastroenterol 1987;22:124-8.

13. Han-Shiang C. Curative resection of colorectal adenocarcinoma: multivariate analysis of 5-year follow up. World J Surg 1999;23:1301-6.
14. Hermanek P, Guggenmoos-Holzmann I, Gall FP. Prognostic factors in rectal carcinoma. A contribution to the further development of tumor classification. Dis Colon Rectum 1989;32:593-9.

15. Koch M, McPherson TA, Egedahl RD. Effect of sex and reproductive history on the survival of patients with colorectal cancer. J Chron Dis 1982;35:69-72.

16. Kune GA, Kune S, Field B, White R, Brough W, Schellenberg R, Watson LF. Survival in patients with large-bowel cancer. A population-based investigation from the Melbourne Colorectal Cancer Study. Dis Colon Rectum 1990;33:938-46.

17. Li F, Kishida T, Kobayashi M. Serum iron and ferritin levels in patients with colorectal cancer in relation to the size, site and disease stage of cancer. J Gastroenterol 1999;34:195-9.

18. Mäkelä J, Kiviniemi H, Laitinen S. Survival after operations for colorectal cancer in patients aged 75 years or over. Eur J Surg 2000;166:473-9.

19. Mulcahy HE, Patchett SE, Daly L, O'Donoghue DP. Prognosis of elderly patients with large bowel cancer. Br J Surg 1994;81:736-8.

20. Nelson RL, Persky V, Turyk M. Determination of factors responsible for the declining incidence of colorectal cancer. Dis Colon Rectum 1999;42:741-52.

21. Newland RC, Dent OF, Lyttle MNB, Chapuis PH, Bokey EL. Pathologic determinants of survival associated with colorectal cancer with lymph node metastases. Cancer 1994;73:2076-82.

22. Nicholls RJ, Mason AY, Morson BC, Dixon AK, Fry IK. The clinical staging of rectal cancer. Br J Surg 1982;69:404-9.

23. Obrand DI, Gordon PH. Incidence and patterns of recurrence following curative resection for colorectal carcinoma. Dis Colon Rectum 1997;40:15-24.

24. Scott NA, Wieand HS, Moertel CG, Cha SS, Beart RW, Lieber MM. Colorectal cancer. Dukes' stage, tumor site, preoperative plasma CEA level, and patient prognosis related to tumor DNA ploidy pattern. Arch Surg 1987;122:1375-9.

25. Shepherd JM, Jones JSP. Adenocarcinoma of the large bowel. Br J Cancer 1971;25:680-90.

26. Sitzler PJ, Seow-Choen F, Ho YA, Leong APK. Lymph node involvement and tumor depth in rectal cancers. An analysis of 805 patients. Dis Colon Rectum 1997;40:1472-6.

27. Slattery ML, Friedman GD, Potter JD, Edwards S, Caan BJ, Samowitz W. A description of age, sex, and site distributions of colon carcinoma in three geographic areas. Cancer 1996;78:1666-70.

28. Spratt JS Jr, Spjut HJ. Prevalence and prognosis of individual clinical and pathologic variables associated with colorectal carcinoma. Cancer 1967;20:1976-85. 
29. Steinberg SM, Barwick KW, Stablein DM. Importance of tumor pathology and morphology in patients with surgically resected colon cancer. Cancer 1986;58:1340-5

30. Tang R, Wang JY, Chen JS, Chang-Chien CR, Tang S, Lin SE, You YT, Hsu KS, Ho YS, Fan HA. Survival impact of lymph node metastasis in TNM stage III carcinoma of the colon and rectum. J Am Coll Surg 1995;180:705-12.

31. Vanek VW, Whitt CL, Abdu RA, Kennedy WR. Comparison of right colon, left colon, and rectal carcinoma. Am Surg 1986;52:504-9.

32. Wiggers T, Arends JW, Volovics A. Regression analysis of prognostic factors in colorectal cancer after curative resections. Dis Colon Rectum 1988;31:3341 .
33. Wolmark N, Wienand S, Rockette HE, Fisher B, Glass A, Lawrence W, Lerner H, Cruz AB, Volk H, Shibata H, Evans J, Prager D. The prognostic significance of tumor location and bowel obstruction in Dukes B and C colorectal cancer. Ann Surg 1983;198:743-52.

34. Wolters U, Stützer H, Keller HW, Schröder U, Pichlmaier H. Colorectal cancer a multivariate analysis of prognostic factors. Eur J Surg Oncol 1996;22:592-7.

35. Yamamoto S, Mochizuki H, Hase K, Yamamoto T, Ohkusa Y, Yokoyama S, Ushitani Y, Tamakuma S. Assessment of clinicopathologic features of colorectal mucinous adenocarcinoma. Am J Surg 1993;166:257-61.

Recebido em $8 / 2 / 2002$ Aprovado em 29/8/2002. 